\title{
Simulating the Mechanism of Antimicrobial Lipopeptides with All- Atom Molecular Dynamics
}

\author{
Joshua N. Horn, Tod D. Romo, and Alan Grossfield* \\ Department of Biochemistry and Biophysics, University of Rochester Medical Center, Rochester, New York 14642
}

\begin{abstract}
The emergence of antibiotic resistant pathogens is one of the major medical concerns of the 21 st century, prompting renewed interest in the development of novel antimicrobial compounds. Here we use microsecond-scale all-atom molecular dynamics simulations to characterize the structure, dynamics, and membrane-binding mechanism of a synthetic antimicrobial lipopeptide, C16-KGGK. Our simulations suggest that these lipopeptides prefer to aggregate in solution and alter the intrinsic order of the lipid bilayer upon binding. From these results and previous coarse-grained simulations, we have developed a simple model for the binding and insertion process for these lipopeptides.
\end{abstract}

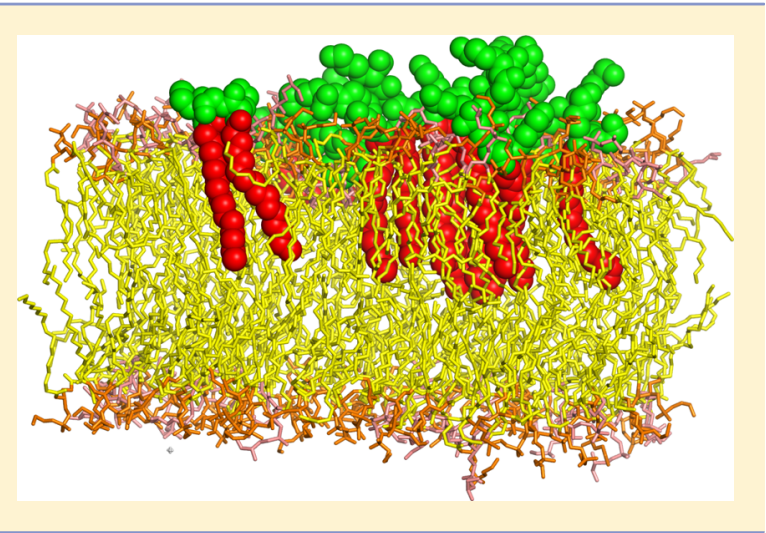

Antibiotic-resistant bacteria and fungi are a major health concern worldwide, driving research into the development of novel classes of antibiotics. One such promising class, peptides with antibacterial and antifungal properties, is a leading candidate as a scaffold for future antibiotics. ${ }^{1}$ These antimicrobial peptides, or AMPs, serve as innate immune system components found in all multicellular organisms. ${ }^{2}$ First isolated from insects ${ }^{3}$ and tree-frogs, ${ }^{4}$ they are noteworthy for their potency against bacteria and fungi. ${ }^{5,6}$ Membrane-active AMPs tend to share a common set of characteristics, specifically an amphipathic structure and a net positive charge. ${ }^{7}$ AMPs target the specific compositions of bacterial membranes, making them less vulnerable to evolved resistance than traditional "single binding site" antibiotics. ${ }^{8}$ However, their physical mechanisms for targeting and permeabilizing lipid bilayers are varied, and in many cases unknown. In general, their net positive charge is assumed to provide the selectivity for bacterial membranes, which tend to have large concentrations of negatively charged lipids, while their amphipathic structure allows them to bind to the lipid bilayer. A number of models for antimicrobial action have been suggested, including poration, ${ }^{9}$ detergent permeabilization, ${ }^{10}$ and membrane destabilization after surface coating. ${ }^{11}$ There appears to be no single mechanism for all AMP action; rather, any given AMP species may function by some subset of these models (depending on the lipid composition and peptide concentration) or even other unproposed models.

Despite a few successes, such as daptomycin ${ }^{12}$ and other promising peptide antibiotics in clinical trials, ${ }^{13}$ natural AMPs are generally not good drug candidates. Peptides containing 10-20 amino acids are far larger than typical drug-like compounds and tend to be prohibitively expensive to synthesize in useful quantities. They also suffer from issues of bioavailability: peptidases degrade free peptides in the bloodstream. ${ }^{14}$ To overcome these limitations, the Shai laboratory designed smaller synthetic molecules with properties similar to naturally occurring AMPs. They demonstrated that conjugating aliphatic acids to the N-terminus of AMPs can bestow selectivity and potency for microbial pathogens. ${ }^{15,16}$ In particular, they focused on a set of relatively potent ultrashort antimicrobial lipopeptides, which they called USLiPs, built around a common architecture: a four-residue peptide with two lysines, a net positive charge, at least one D-amino acid, and a 16-carbon fatty acid attached to the N-terminus. ${ }^{17}$ While these molecules have promising antibacterial properties, it appears that their mode of action is different than traditional AMPs, largely due to dramatic differences in structure: USLiPs are detergent-like in structure and bear little resemblance to the active folds of known AMPs. ${ }^{18}$ For this reason, it is important to gain a better atomic-level understanding of their interactions with lipid bilayers.

Previous coarse-grained molecular dynamics simulations of the most potent of these USLiPs, C16-KGGK (bold indicates a D-enantiomer), provided some insights into a possible mechanism for antimicrobial action. ${ }^{19}$ In that work, lipopeptide aggregates partially demixed the membrane by attracting the anionic lipid species. This feature has been noted for some other AMPs and may play a role in inhibiting bacterial growth by a number of proposed means: by creating boundary defects between domains, altering membrane curvature, ${ }^{20}$ changing membrane polarization, ${ }^{21,22}$ or reducing the stability of previously formed lipid domains. ${ }^{23}$ This in turn could disrupt the cell's ability to sort proteins in the membrane, or inhibit those proteins' function. Binding of charged peptides also alters

Received: June 17, 2013

Revised: July 18, 2013

Published: July 22, 2013 
the membrane environment by changing the local concentration of free salt and even the transmembrane voltage.

While these results were interesting, limitations in the coarsegrained model have led us to further test this system using more detailed all-atom simulations. In this study, we explore the effects of the binding and insertion of C16-KGGK molecules on bilayer structure and organization using an ensemble of microsecond-scale all-atom molecular dynamics. We have characterized the binding process of C16-KGGK, noting critical contacts formed during this process.

\section{METHODS}

Simulation Protocol. Our simulations were run with NAMD version 2.6 and the $\mathrm{NP} \gamma \mathrm{T}$ ensemble, with constant particle number, pressure, surface tension, and temperature. For the protein, we used the CHARMM22 force field, ${ }^{24}$ with the CMAP backbone torsions; ${ }^{25,26}$ to handle the D-amino acids, we created new backbone atom types with identical parameters, except for the CMAP term, where we transposed the matrix. For lipids, we used the CHARMM27 force field, with the refined CHARMM parameters for saturated chains ${ }^{27}$ and polyunsaturated chains. ${ }^{28}$ Langevin dynamics was used for all heavy atoms, with the temperature set to $300 \mathrm{~K}$. Construction and equilibration were performed with CHARMM version $34 .^{29}$ Long-range electrostatics were calculated using smooth particle-mesh Ewald summation ${ }^{30}$ with a $96 \times 96 \times 96$ grid. van der Waals interactions were smoothly cutoff from 9 to $10 \AA$ with the pairlist maintained out to $12 \AA$. A 2 fs time step was used, with bonds constrained to their equilibrium lengths using RATTLE. $^{31}$ All analysis was done at 100 ps resolution unless otherwise noted.

System Construction. Systems were built using the CHARMM package by randomly scattering lipopeptides on one side of a pre-equilibrated bilayer and then solvating the system. The result was thoroughly minimized and then equilibrated via a cycle of alternating minimization and very short dynamics run, starting with the system at a temperature of $50 \mathrm{~K}$ and incrementally increasing the temperature until the final temperature of $300 \mathrm{~K}$ was achieved. The bilayers were constructed using a previously described library-based proto$\mathrm{col}^{32}$ and run for at least $100 \mathrm{~ns}$ prior to use to ensure that the area per lipid and other physical quantities had time to equilibrate. We chose to simulate a 2:1 POPE/POPG bilayer, which serves as a "gram-negative bacteria-like" model composition.

Table 1 shows all simulation systems, details, and run times. In general, the simulations fall into two general categories: binding systems and high concentration systems. The binding systems were designed to mimic the physiological situation, where the lipopeptides all bind to one membrane leaflet, as if approaching from outside the cell. To achieve this effect, multiple simulations were run but only those where all lipopeptides bound to the same leaflet were continued to longer time scales (and only these are reported). For the high concentration systems, 60 lipopeptides were allowed to bind, but they were free to bind to either leaflet (the solution was crowded with lipopeptides, making it difficult for all of the lipopeptides to bind to the same leaflet).

We began by systematic testing of the bilayer at different tensions in order to ensure that our system equilibrated to the correct area per lipid, as this property likely affects the binding and insertion of lipopeptides, and initially settled on the use of a tension of $(\gamma)$ of $27.5 \mathrm{dyn} / \mathrm{cm}$. However, longer runs showed
Table 1. Table of All Simulations and Details ${ }^{a}$

\begin{tabular}{|c|c|c|c|}
\hline system & tension & lipopeptides & length (ns) \\
\hline \multirow[t]{3}{*}{ binding high tension } & 35 & 20 & 1986 \\
\hline & & & 2002 \\
\hline & & & 1720 \\
\hline \multirow[t]{4}{*}{ binding low tension } & 27.5 & 20 & 1529 \\
\hline & & & 1535 \\
\hline & & & 1509 \\
\hline & & & 1554 \\
\hline \multirow[t]{3}{*}{ high conc high tension } & 35 & 60 & 1103 \\
\hline & & & 403 \\
\hline & & & 330 \\
\hline \multirow[t]{4}{*}{ high conc low tension } & 27.5 & 60 & 178 \\
\hline & & & 178 \\
\hline & & & 136 \\
\hline & & & 134 \\
\hline \multirow[t]{3}{*}{ neat } & 35 & 0 & 92 \\
\hline & & & 69 \\
\hline & & & 64 \\
\hline \multirow[t]{3}{*}{ neat } & 27.5 & 0 & 565 \\
\hline & & & 714 \\
\hline & & & 556 \\
\hline
\end{tabular}

${ }^{a}$ All systems include 180 lipids in a ratio of 2:1 POPE/POPG lipids. Tensions are in dyn $/ \mathrm{cm}$.

that this tension yielded bilayers with areas per lipid below experimental expectation. As a result of this lower area, the bilayer was tightly packed, and the calculated ${ }^{2} \mathrm{H}$ parameters were higher than those typically reported from experiment. However, an applied tension of $35 \mathrm{dyn} / \mathrm{cm}$ maintained bilayers with values for the area per lipid in agreement with experiment (data not shown). We analyzed both the high and low tension simulations; in most cases, the lipopeptide-bilayer interactions appeared qualitatively insensitive to tension. Recently, an update to the CHARMM lipid force field - CHARMM36 - was released, after we began this project. ${ }^{33}$ This published work focused on PC headgroups, but preliminary testing (data not shown) suggested that POPE and mixed POPE/POPG bilayers still require a tension to retain reasonable areas per lipid. We are in the process of running a thorough titration of tensions to find the ideal tension before we run future simulations with the new forcefield.

Simulation Analysis. All analysis was performed with tools developed with Lightweight Object Oriented Structure (LOOS) analysis library. ${ }^{34}$ LOOS is an object-oriented library implemented in $\mathrm{C}++$ and Boost and accessible to Python that provides functionality for creating new tools for the analysis of molecular dynamics simulations. LOOS is available for download at http://loos.sourceforge.net.

Fractional Contacts Analysis. To assess the nature of the environment surrounding C16-KGGK, we used a fractional contact analysis. For each lipopeptide, we counted every heavy atom within a $4 \AA$ radius of a lipopeptide heavy atom and reported what fraction of those atoms were from other lipopeptides, each lipid type, and water. We ignored ions, as they make up very few of the total number of atoms in the system.

To quantify the order in which the interactions between lipopeptides and the lipid bilayer form, another fractional contact calculation was performed. This time, the calculation measured the fraction of neighboring atoms that were lipid or water, for each of the following lipopeptide components: the D- 
lysine, the standard lysine, and the palmitoyl chain. Ions were again ignored in the calculation, as were other lipopeptides.

Lateral Radial Distribution Function. To assess the lateral structure of the bilayer, we computed the two-dimensional radial distribution function (RDF) of various lipid species relative to lipopeptides. Each molecule was treated as a single point, located at its centroid, and the RDF was computed as

$$
g\left(r_{x y, i}\right)=\frac{1}{N_{\text {pair }} \pi\left\{\left(r_{x y, i}+\frac{\delta}{2}\right)^{2}-\left(r_{x y, i}-\frac{\delta}{2}\right)^{2}\right\}}\left\langle n_{\text {pair }}\left(r_{x y, i}\right)\right\rangle
$$

where $r_{x y, i}$ is the distance in the plane of the membrane at the center of the $i$ th bin, $N_{\text {pair }}$ is the number of pairs possible (equal to $N_{\mathrm{a}} N_{\mathrm{b}}$ if a and $\mathrm{b}$ are different chemical species, $N(N-1) / 2$ if the RDF is for a single chemical species to itself), $\delta$ is the width of the histogram bins, and $n_{\text {pair }}\left(r_{x y, i}\right)$ is the number of pairs found in distances belonging to bin $i$ in any given trajectory snapshot. We also tracked the time evolution of the RDF, splitting the trajectory into $1 \mathrm{~ns}$ windows.

Aggregates. To quantify lipopeptide aggregation, we identified which pairs of lipopeptides were in contact with one another - a pair of lipopeptides was considered an aggregate if they had at least four heavy atom-heavy atom contacts within $3 \AA-$ and then used this connectivity information to define the aggregates.

Order Parameters. The average orientation of the acyl $\mathrm{C}-\mathrm{H}$ bonds relative to the membrane normal can be expressed by the order parameter:

$$
S_{\mathrm{CD}}=-\frac{1}{2}\left\langle 3 \cos ^{2} \theta_{\mathrm{CD}}-1\right\rangle
$$

This quantity is proportional to the quadrupolar splitting seen in ${ }^{2} \mathrm{H}$ NMR spectra, which can be readily measured from the spacing of the doublet peaks. However, because all acyl deuterons have essentially the same resonance frequency and the experiments are usually done with perdeuterated lipids, the experiments cannot resolve which doublet peaks are due to which positions along the fatty acid chain. Instead, experimental results are by convention plotted with the $S_{\mathrm{CD}}$ values dropping monotonically along the chain. With simulations, we can easily discern which carbon along the acyl chain is responsible for each $S_{\mathrm{CD}}$ value and report them without any sorting.

\section{RESULTS AND DISCUSSION}

Lipopeptides Bind and Insert into Membrane. In solution, the lipopeptides rapidly aggregate into a loose micellelike structure with the fatty acid chains largely desolvated, which makes sense given their detergent-like structure. Aggregation occurs concurrently with association with the bilayer surface. However, it is likely that the two processes are not related; rather, the initial states were constructed with the lipopeptides close to the lipid bilayer (shown in Figure 1). It appears that these aggregates satisfy some of the hydrophobic packing preferences of the lipopeptide tails, as they can persist for many tens or even hundreds of nanoseconds. However, the micelles do not completely shield the acyl chains from water, so eventually the lipopeptides switch to a fully inserted state, with the acyl chains buried in the membrane's hydrophobic core and the peptide portion free to interact with water and the lipid headgroups. In each trajectory, full insertion of all lipopeptides occurred in under a microsecond, and there were no dissociation events.

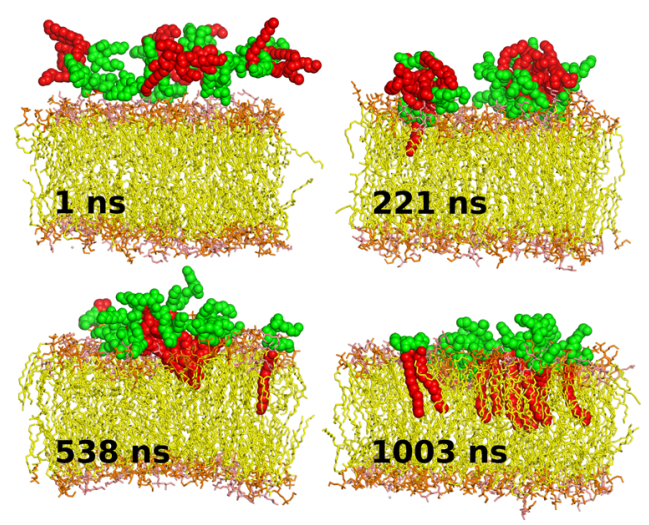

Figure 1. Trajectory snapshots from one of the high-tension binding simulations with 20 lipopeptides. Lipids are yellow and salmon for tail and headgroup, respectively. The peptide portion of the lipopeptides is in green, and the hydrocarbon tails are in red.

To quantify the binding and insertion process, we began by looking at the average distance of the lipopeptides' centroid (taken as a group) from the bilayer center as a function of time. This is shown in Figure 2 and highlights the concerted insertion

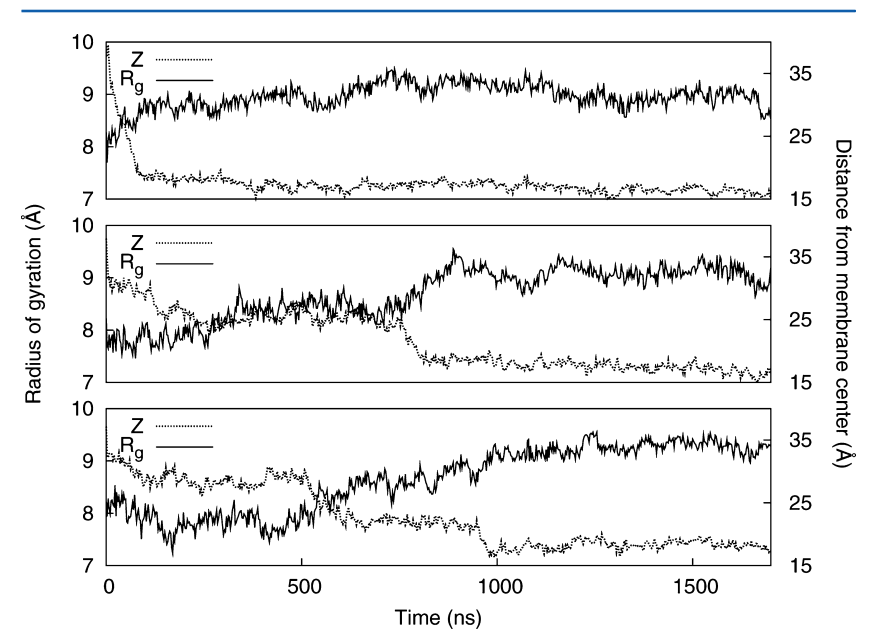

Figure 2. Average lipopeptide radius of gyration (solid) and the average distance of centroid of each lipopeptide from the membrane center (dots) against time for three high-tension binding trajectories.

of clusters of lipopeptides (shown by stepwise drops in the curve), until all of the lipopeptides are inserted, and the average distance from the membrane center is about $18 \AA$. In the first trajectory, this insertion is rapid and occurs early in the simulation, with all of the lipopeptides fully inserted in the first $100 \mathrm{~ns}$. The process is more gradual in the second trajectory, with one lipopeptide micelle persisting at the membrane surface for roughly $750 \mathrm{~ns}$.

The structure of the lipopeptides changes during the insertion process. This can be most easily demonstrated by calculating the average radius of gyration for the lipopeptides as a function of time. This quantity is also shown in Figure 2 to highlight its correlation with the lipopeptide position. As lipopeptides insert into the bilayer, the average radius of gyration tends to increase, in a similarly stepwise manner; this is due mostly to changes in the acyl chain portion, which elongates to match the structure of the surrounding lipid matrix. 
Bilayer Binding Interactions. To quantify the binding process, we calculated the fraction of neighboring atoms that are lipid atoms and water atoms for each part of the lipopeptides. The average lipid fraction for the lysines, Dlysines and the palmitoyl chains for one representative trajectory is shown in Figure 3. Contacts between the two

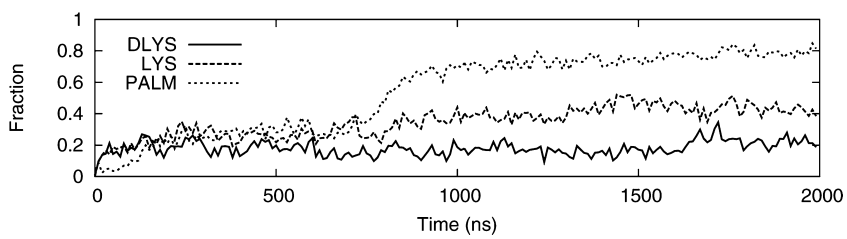

Figure 3. Fraction of neighboring contacts that are lipid atoms for each of the lysines and the palmitoyl chain for one representative hightension binding trajectory. The remaining fraction for each curve is water. Data are plotted every $10 \mathrm{~ns}$ for clarity.

lysines and the lipids increase first in the binding process, suggesting that electrostatic attraction is the initial driving force for association with the protein surface; this is consistent with prior simulations of a different lipopeptide, C6-LfB6. ${ }^{35}$ After the lysines make contact with the lipids, the palmitoyl chain inserts into the bilayer, as indicated by a dramatic increase of lipid contact (close to 0.8). In the trajectory shown, this increase is stepwise, with the first increase between 50 and 100 ns, and the second between 700 and 800 ns. These correspond with the insertion of the two major clusters of lipopeptides. As the palmitoyl chains insert, the lipid contacts with the D-lysines remain constant, while the contacts with the L-lysines increase. This demonstrates lipopeptide positioning within the bilayer: the palmitoyl chain is embedded deeply into the hydrophobic core of the bilayer, the L-lysines have high contact to both solution and the bilayer as they interact within the headgroup region, and the terminal D-lysines are well hydrated and likely exist mostly in solution further from the bilayer. This ordering is the result of sequence; the L-lysine is the $\mathrm{N}$-terminal residue, close to the hydrophobic tail, while the $\mathrm{D}$-lysine is at the Cterminus. We do not believe the chirality of the lysines has any effect on its hydration.

We also computed, for each lipopeptide, the fraction of contacts to each lipid species, other lipopeptides, and water. Figure 4 shows this data for one trajectory (the same one used

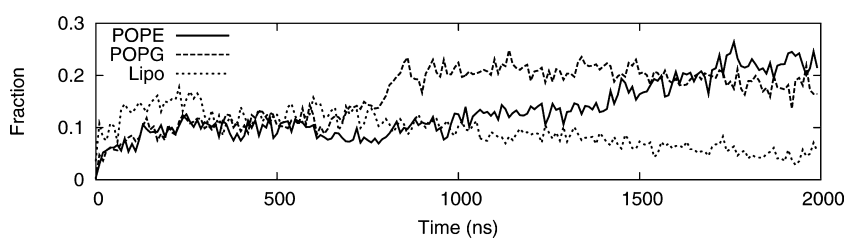

Figure 4. Fraction of contact of lipopeptides with POPE, POPG, and other lipopeptides over time for one high-tension binding trajectory. Contact with water begins at 1 , as all lipopeptides begin in solution, but drops as the lipopeptides associate with the bilayer. Data are plotted every $10 \mathrm{~ns}$ for clarity.

in Figure 3), with the water fraction omitted for clarity. In all of the trajectories, the lipopeptide-lipopeptide interactions increase significantly over the first $200 \mathrm{~ns}$ or so, as lipopeptides free in solution quickly associate with one another. However, the lipopeptide-lipopeptide contacts decrease steadily over the rest of the trajectory, as the lipopeptides first insert into the membrane and then disperse laterally. Interestingly, the fraction of contacts to POPG and POPE is similar throughout the trajectory, with POPG at greater fractions for periods. Given that the concentration of POPE is twice that of PG, this suggests a significant preference for the anionic lipid.

Lipid Preferences and Aggregation. To better understand the effects of lipopeptides on membrane organization, we computed the lateral radial distribution functions (RDF) of various bilayer components about the lipopeptides. These data are shown in Figure 5; for the purposes of comparison, we

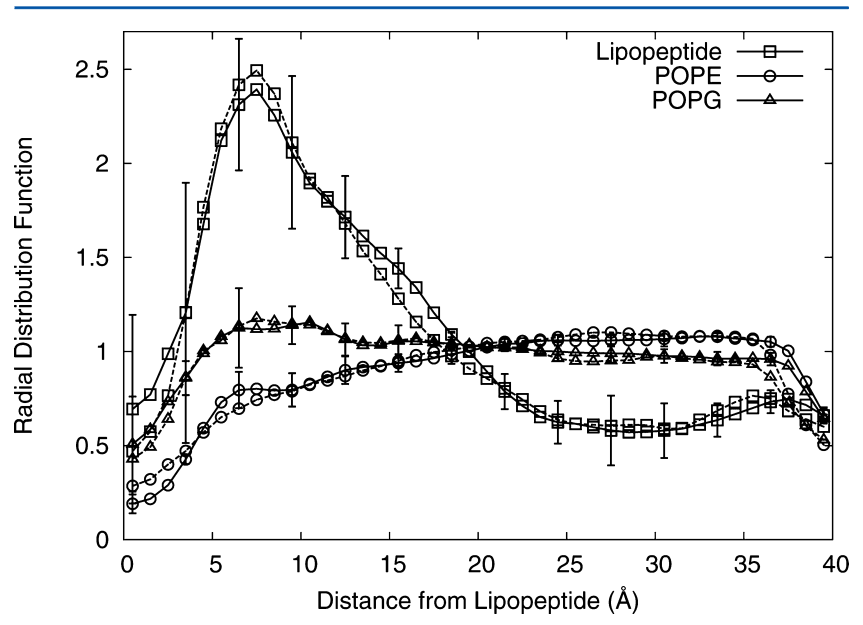

Figure 5. Lateral radial distribution function of POPE (circle), POPG (triangle), and other lipopeptides (square) as a function of distance from each lipopeptide. Data are shown for both high-tension (solid) and low-tension (dashed) runs, with the first $500 \mathrm{~ns}$ excluded to ensure that all lipopeptides are completely inserted. The error-bars reflect the deviation among the high-tension runs (the deviation is very similar for the low-tension runs).

show both the low and high tension runs. The most notable feature is the dramatic lipopeptide-lipopeptide peak around 7 $\AA$, which reflects the aggregation noted earlier. There is also a lipopeptide-POPG peak between 5 and $10 \AA$, while POPE does not reach bulk levels until nearly $20 \AA$ away from the lipopeptides. This demonstrates that POPG tends to be enriched at distances close to the lipopeptides, while POPE is excluded from this short-range interaction. These preferences are likely the result of electrostatic interactions: cationic lipopeptides are attracted to anionic lipids. This supports the idea that the selectivity of cationic antimicrobial molecules for bacterial membranes is due to their significantly higher concentration of anionic lipids.

Because the simulation begins far from equilibrium, with the lipopeptides isolated in the solvent, computing simple averages can be deceptive. For this reason, we track the timedependence of the RDFs as well, shown in Figure 6. In the first panel, the lipopeptide-lipopeptide RDF shows the same peaks at short-range that have already been shown. However, after a microsecond of simulation, this peak begins to disappear and is nearly gone by the end of the simulation, replaced by even density at a long distance. All of this suggests that while lipopeptides prefer to aggregate in solution and maintain their oligomerization as they bind, they will begin to dissipate and spread laterally across the membrane once completely inserted; there is no evidence for stable peptide oligomerization in the fully inserted state. This in turn suggests that the mechanism of 


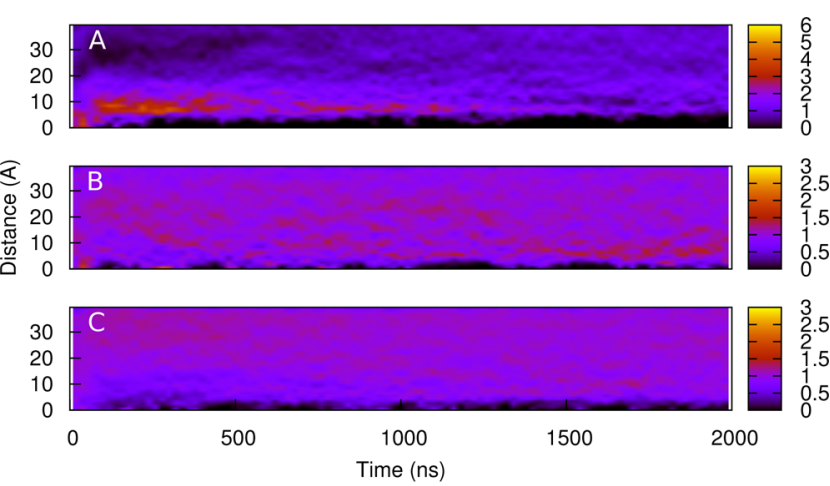

Figure 6. Time-dependent radial distribution functions for lipopeptide-lipopeptide (A), lipopeptide-POPG (B), and lipopeptide-POPE (C) for one high-tension trajectory. For clarity, the lipopeptide-lipopeptide density is shown on a different scale.

the lipopeptides studied here differs significantly from that of other commonly studied AMPs, such as alamethicin or melittin.

The second and third panels of Figure 6 show the timedependent RDFs for lipopeptide-POPG and lipopeptidePOPE interactions, respectively. The interactions with lipids at ranges shorter than $10 \AA$ away increase as the simulation progresses, as would be expected as the lipopeptidelipopeptide clusters disperse. This change is more noticeable for the POPG lipid, indicating that the lipopeptides' preference for POPG are long-lived and persist even after lipopeptide dispersion. The dispersion of the lipopeptides is also shown in Figure 7, where we plot the number of lipopeptide aggregates in our systems as a function of time. The number of aggregates steadily increases throughout the trajectories, again suggesting that the lipopeptides are still in the process of dispersing from the "clumped" state formed in the solution phase.

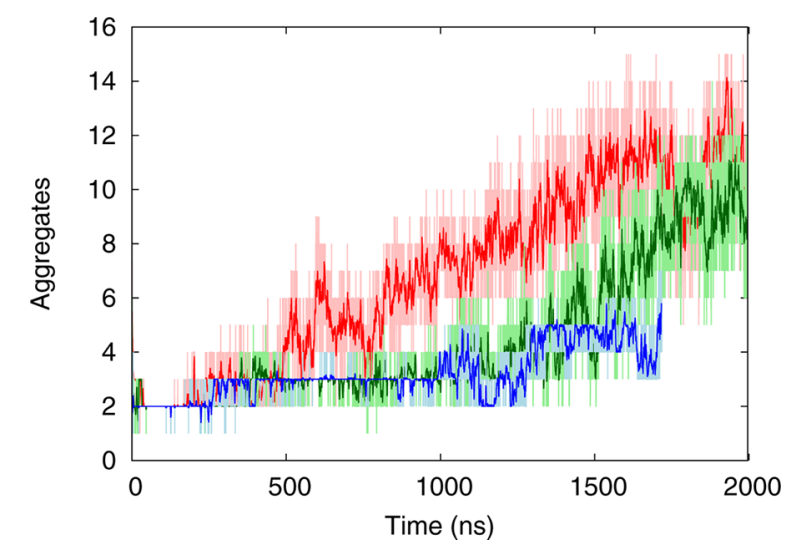

Figure 7. Number of aggregates of lipopeptides in the simulation over time for the three high-tension runs. The pale lines are the raw, discrete data, while the dark lines are windowed 2 ns averages.

Lipopeptides Alter Bilayer Order. Using the calculated acyl chain order parameter profile, we can quantify the impact of the lipopeptides on bilayer structure. In Figure 8, we show the average order parameters for lipids in both the lipopeptidebound and unbound leaflets for each of the high-tension and low-tension binding systems, as well as for the palmitoyl chain of the lipopeptides in those systems. For this analysis, the first microsecond was omitted to focus only on the part of the trajectory where the lipopeptides were fully bound and inserted.

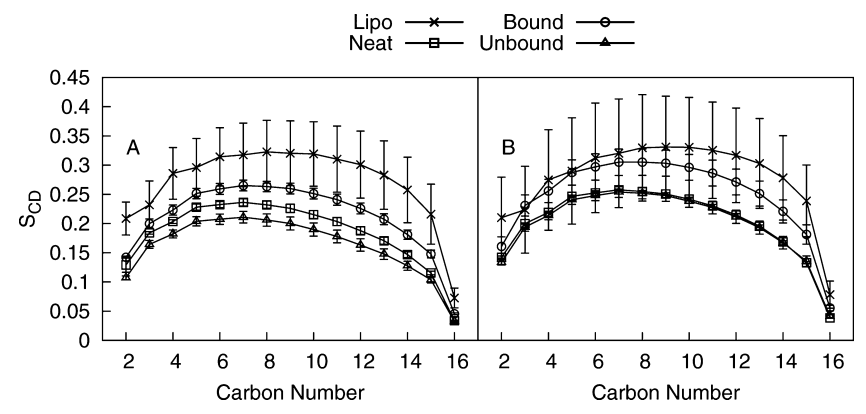

Figure 8. Order parameters for the palmitoyl chains of the bound and unbound leaflets, as well as of the lipopeptide tails, from the (A) hightension and (B) low-tension trajectories. "Neat" indicates the order parameters from an equivalent system in the absence of lipopeptides. The error-bars indicate the standard deviation among the trajectories.

As a control, the order parameters for lipopeptide-free neat systems were included.

All of the order parameter curves show the same characteristic shape, with lower order associated with the carbons at both ends of the chain and higher order the middle. Lower order for carbons 2 to 4 is the result of the consistent tilt found in the chain as it connects to the glycerol backbone. The order peaks between carbons 6 and 8, as the chain "straightens out", and decreases again as the terminal carbons display greater flexibility and range of motion.

The differences between the high and low-tension systems are significant, with the low-tension simulations consistently more ordered as expected, but the qualitative trends are the same. The lipopeptide acyl chains are more ordered than the surrounding lipid matrix, as we would expect for a single chain without the restrictions that lipids with two tails face.

One interesting phenomenon is that the presence of the lipopeptides increases the average order parameters for the leaflet they are bound to. This effect is partially due to the limitations of utilizing a periodic boundary; both leaflets have the same number of lipids and the same area, but the excluded volume from the lipopeptides reduces the area available to lipids.

However, this is not the sole reason for this effect. In the longest of the high-concentration runs, the lipopeptides bound asymmetrically, with 20 lipopeptides in one leaflet, 30 in the other, and the remaining 10 associated with the surface. The leaflet with 30 lipopeptides had higher order parameters than the one with 20, but both leaflets were more ordered than the neat system. This again suggests that the biological effects of the lipopeptides may not arise from locally disordering the membrane to induce leakage; rather, the binding may instead bend or otherwise alter the properties of the membrane. Membrane thickening has been observed experimentally with other AMPs. $^{36}$

Reconciling Multiscale Simulations. In previous work, we employed coarse-grained models to characterize the binding of a micelle of C16-KGGK molecules to model bilayers. ${ }^{19}$ In that work, the system often formed a metastable state where the micelle remained intact at the membrane surface without inserting. Instead, the micelle attracted and ordered a block of anionic lipids. We hypothesized that this demixing could be part of the physiological antibacterial mechanism, since altering the mixing properties could impact cellular processes that depend on specific lipid compositions. 
The present simulations, at higher resolution but for shorter time scales, are largely consistent with these results. For example, both models predict that the lipopeptides will rapidly aggregate into micelles in solution. In the coarse-grained models, we took advantage of this in our initial system construction and used previously equilibrated micelles as the starting point for the production simulations. For the all-atom simulations, we generally worked with a smaller number of lipopeptides and performed all simulations in the presence of the membrane. As a result, the micelles form in competition with membrane binding and are not as stable or well-formed as those in the coarse-grained simulations. On the other hand, we believe that much of the stability of the surface-bound micellar state in those simulations is an artifact of the coarse-grained system's limited electrostatic model. For this reason, we instead focused on the effects of lipopeptides through insertion and dispersion.

The most appropriate comparison for our simulations is to the lone coarse-grained simulation of micelle binding that resulted in complete insertion. In that system, as in the current all-atom trajectories, the micelle inserted into the lipid bilayer and the lipopeptides gradually dispersed. Preferential interactions with POPG lipids at short-range were maintained, resulting in a well-mixed bilayer with each lipopeptide paired with a few POPG lipids. These interactions are primarily electrostatic: insertion satisfies the hydrophobic interactions of the lipopeptide tails, leaving cationic peptides to repel one another and interact with the anionic lipids.

Despite the similarities, the presented all-atom trajectories provide information that cannot be gathered easily from much simpler coarse-grained models. The most notable is the change in order parameters that results from lipopeptide binding. While measures of chain order can be calculated from coarsegrained models, they are not directly comparable to deuterium order parameters determined from solid-state NMR. Quantitatively, these parameters depend on the applied tension of the bilayer, but the trends hold across both tensions simulated.

Model for Binding and Interactions. The combination of coarse-grained and all-atom simulations allow us to construct a model for the interaction between C16-KGGK and lipid bilayers. In solution, the lipopeptides form micelles or higher order structures (consistent with experiment ${ }^{37}$ ), which helps improve their bioavailability. The micelles diffuse in solution until they approach a membrane, where they form a surfacebound complex and attract anionic lipids. On a longer timescale, the lipopeptides will insert into the bilayer and gradually disperse. By binding in an aggregate form, the lipopeptides retain much higher local concentrations. Upon eventual insertion, this would cause lipopeptide effects on bilayer structure, including changes to order and curvature, to be more dramatic and damaging.

\section{CONCLUSIONS}

Our previous coarse-grained simulations gave us a model for C16-KGGK interactions with the bilayer that involved aggregation and bilayer reorganization. ${ }^{19}$ However, the limitations inherent in the coarse-grained models used convinced us to complement this work with microsecondscale all-atom simulations. We chose not to use prebuilt micelles, both to reduce the simulation size and to more thoroughly explore the effect of full inserted lipopeptides, a process that would likely occur slowly in simulations started from micelles.
The first thing we saw was the clear preference for aggregation of the lipopeptides when in solution, driven by the lipopeptides' hydrophobic tails. As the lipopeptides aggregated, they also interacted with the bilayer; the cationic lysines in the peptide segment made preferential contact with the anionic headgroups of the POPG lipids. However, the surface-bound aggregates did not totally protect the lipopeptide acyl chains from water, so over time the lipopeptides inserted into the membrane. In the fully inserted state, the acyl chains are fully buried in the core of the membrane, while the peptide portion remained largely hydrated, while also making contact with the headgroups.

After insertion, the aggregates fall apart and the lipopeptides disperse laterally across the surface of the membrane. The presence of lipopeptides increases the order of the hydrocarbon lipid tails, an effect that occurs in systems with higher concentrations of lipopeptides and binding to both leaflets, leading us to conclude that it is not based solely on the asymmetric increase of area in one leaflet relative to the other.

All of this, coupled with previously published coarse-grained results, ${ }^{19}$ suggests a possible model for function: the lipopeptides achieve their bacteriocidal effect through surface aggregation and lipid demixing, and after complete insertion through increased bilayer ordering. In both cases, the mode of action differs from either the classical poration models or more recent carpet models of antimicrobial peptide action. ${ }^{11}$ Similar effects have been seen for some cell-penetrating peptides, specifically the importance of lysine-headgroups interactions and induced changes to the bilayer order. ${ }^{38}$

This model is related to the "interfacial activity model" that has been explored by the Wimley laboratory when developing new AMPs ${ }^{39,40}$ and has been described in some detail elsewhere. ${ }^{41}$ Essentially, it considers the ability of a peptide to partition into a membrane at the bilayer-water interface and affect the organization of the lipids. There is a balance and interplay between lipopeptide size, amphipathicity, and electrostatics, along with environmental factors such as lipid composition, lipid packing, and salt concentration. For our system, the interfacial activity seems to be affected and even enhanced by the aggregation properties of the lipopeptide.

\section{AUTHOR INFORMATION}

\section{Corresponding Author}

*E-mail: alan_grossfield@urmc.rochester.edu.

\section{Funding}

This work was supported by GM095496 (A.G.) and GM068411 from the Institutional Ruth L. Kirschstein National Research Service Award (J.N.H.).

\section{Notes}

The authors declare no competing financial interest.

\section{ACKNOWLEDGMENTS}

We thank IBM for access to the BlueGene/L supercomputer at the T.J. Watson Research Center used to generate these simulations. Thanks also go to the Center for Integrated Research Computing at the University of Rochester for providing computational resources for the mentioned coarsegrained simulations. We thank Dr. Alexander Vogel for helpful discussions and experimental consultation. 


\section{ABBREVIATIONS}

MD, Molecular Dynamics; AMP, antimicrobial peptides; RDF, radial distribution function; POPE, palmitoyl-oleoyl-phosphatidylethanolamine; POPG, palmitoyl-oleoyl-phosphatidylglycerol

\section{REFERENCES}

(1) Koczulla, A. R., and Bals, R. (2003) Antimicrobial peptides: current status and therapeutic potential. Drugs 63, 389-406.

(2) Boman, H. G. (1995) Peptide antibiotics and their role in innate immunity. Annu. Rev. Immunol. 13, 61-92.

(3) Boman, H. G., and Steiner, H. (1981) Humoral immunity in Cecropia pupae. Curr. Top. Microbiol. Immunol. 94-95, 75-91.

(4) Zasloff, M. (1987) Magainins, a class of antimicrobial peptides from Xenopus skin: isolation, characterization of two active forms, and partial cDNA sequence of a precursor. Proc. Natl. Acad. Sci., U. S. A. 84, $5449-5453$.

(5) Westerhoff, H. V., Juretić, D., Hendler, R. W., and Zasloff, M. (1989) Magainins and the disruption of membrane-linked free-energy transduction. Proc. Natl. Acad. Sci., U. S. A. 86, 6597-6601.

(6) Mygind, P. H., et al. (2005) Plectasin is a peptide antibiotic with therapeutic potential from a saprophytic fungus. Nature 437, 975-980.

(7) Epand, R. M., and Vogel, H. J. (1999) Diversity of antimicrobial peptides and their mechanism of action. Biochim. Biophys. Acta 1462, $11-28$.

(8) Jenssen, H., Hamill, P., and Hancock, R. E. W. (2006) Peptide antimicrobial agents. Clin. Microbiol. Rev. 19, 491-511.

(9) Brogden, K. A. (2005) Antimicrobial peptides: pore formers or metabolic inhibitors in bacteria? Nat. Rev. Microbiol. 3, 238-250.

(10) Bechinger, B., and Lohner, K. (2006) Detergent-like actions of linear amphipathic cationic antimicrobial peptides. Biochim. Biophys. Acta, Biomembr. 1758, 1529-1539.

(11) Shai, Y., and Oren, Z. (2001) From "carpet" mechanism to denovo designed diastereomeric cell-selective antimicrobial peptides. Peptides 22, 1629-1641.

(12) Woodworth, J. R., Nyhart, E. H., Brier, G. L., Wolny, J. D., and Black, H. R. (1992) Single-dose pharmacokinetics and antibacterial activity of daptomycin, a new lipopeptide antibiotic, in healthy volunteers. Antimicrob. Agents Chemother. 36, 318-325.

(13) Vaara, M. (2009) New approaches in peptide antibiotics. Curr. Opin. Pharmacol. 9, 571-576.

(14) Hancock, R. E. W., and Sahl, H.-G. (2006) Antimicrobial and host-defense peptides as new anti-infective therapeutic strategies. Nat. Biotechnol. 24, 1551-1557.

(15) Avrahami, D., and Shai, Y. (2003) Bestowing antifungal and antibacterial activities by lipophilic acid conjugation to D,L-amino acid-containing antimicrobial peptides: a plausible mode of action. Biochemistry 42, 14946-14956.

(16) Avrahami, D., and Shai, Y. (2004) A new group of antifungal and antibacterial lipopeptides derived from non-membrane active peptides conjugated to palmitic acid. J. Biol. Chem. 279, 12277-12285.

(17) Makovitzki, A., Avrahami, D., and Shai, Y. (2006) Ultrashort antibacterial and antifungal lipopeptides. Proc. Natl. Acad. Sci. U. S. A. 103, 15997-16002.

(18) Mangoni, M., and Shai, Y. (2011) Short native antimicrobial peptides and engineered ultrashort lipopeptides: similarities and differences in cell specificities and modes of action. Cell. Mol. Life Sci., $1-14$

(19) Horn, J. N., Sengillo, J. D., Lin, D., Romo, T. D., and Grossfield, A. (2012) Characterization of a potent antimicrobial lipopeptide via coarse-grained molecular dynamics. Biochim. Biophys. Acta, Biomembr. $1818,212-218$

(20) Arouri, A., Dathe, M., and Blume, A. (2009) Peptide induced demixing in $\mathrm{PG} / \mathrm{PE}$ lipid mixtures: a mechanism for the specificity of antimicrobial peptides towards bacterial membranes? Biochim. Biophys. Acta 1788, 650-659.

(21) Radzishevsky, I. S., Rotem, S., Bourdetsky, D., Navon-Venezia, S., Carmeli, Y., and Mor, A. (2007) Improved antimicrobial peptides based on acyl-lysine oligomers. Nat. Biotechnol. 25, 657-659.
(22) Rotem, S., Radzishevsky, I. S., Bourdetsky, D., Navon-Venezia, S., Carmeli, Y., and Mor, A. (2008) Analogous oligo-acyl-lysines with distinct antibacterial mechanisms. FASEB J. 22, 2652-2661.

(23) Epand, R. M., and Epand, R. F. (2011) Bacterial membrane lipids in the action of antimicrobial agents. J. Pept. Sci. 17, 298-305.

(24) MacKerell, A. D., Jr., et al. (1998) All-atom empirical potential for molecular modeling dynamics studies of proteins. J. Phys. Chem. B $102,3586-3616$.

(25) Mackerell, A. D., Jr, Feig, M., and Brooks, C. L., 3rd (2004) Extending the treatment of backbone energetics in protein force fields: limitations of gas-phase quantum mechanics in reproducing protein conformational distributions in molecular dynamics simulations. J. Comput. Chem. 25, 1400-1415.

(26) MacKerell, A. D., Jr, Feig, M., and Brooks, C. L., 3rd (2004) Improved treatment of the protein backbone in empirical force fields. J. Am. Chem. Soc. 126, 698-699.

(27) Klauda, J. B., Brooks, B. R., MacKerell, A. D., Jr., Venable, R. M., and Pastor, R. W. (2005) An ab initio study on the torsional surface of alkanes and its effect on molecular simulations of alkanes and a DPPC bilayer. J. Phys. Chem. B 109, 5300-5311.

(28) Feller, S. E., Gawrisch, K., and MacKerell, A. D. ., Jr. (2002) Polyunsaturated Fatty Acids in Lipid Bilayers: Intrinsic and Environmental Contributions to Their Unique Physical Properties. J. Am. Chem. Soc. 124, 318-326.

(29) Brooks, B. R. et al. (2009) CHARMM: the biomolecular simulation program. J. Comput. Chem. 30, 1545-1614.

(30) Essmann, U., Perera, L., Berkowitz, M. L., Darden, T., Lee, H., and Pedersen, L. G. (1995) A smooth particle mesh ewald method. J. Chem. Phys. 103, 8577-8593.

(31) Andersen, H. C. (1983) RATTLE: A "velocity" version of the SHAKE algorithm for molecular dynamics calculations. J. Comput. Phys. 52, 24-34.

(32) Grossfield, A., Feller, S. E., and Pitman, M. C. (2006) A role for direct interactions in the modulation of rhodopsin by omega-3 polyunsaturated lipids. Proc. Natl. Acad. Sci., U. S. A. 103, 4888-4893.

(33) Klauda, J. B., Venable, R. M., Freites, J. A., O'Connor, J. W., Tobias, D. J., Mondragon-Ramirez, C., Vorobyov, I., MacKerell, A. D., and Pastor, R. W. (2010) Update of the CHARMM All-Atom Additive Force Field for Lipids: Validation on Six Lipid Types. J. Phys. Chem. B 114, 7830-7843.

(34) Romo, T. D., and Grossfield, A. (2009) LOOS: an extensible platform for the structural analysis of simulations. Conf. Proc. IEEE Eng. Med. Biol. Soc. 2009, 2332-2335.

(35) Romo, T. D., Bradney, L. A., Greathouse, D. V., and Grossfield, A. (2011) Membrane binding of an acyl-lactoferricin B antimicrobial peptide from solid-state NMR experiments and molecular dynamics simulations. Biochim. Biophys. Acta 1808, 2019-2030.

(36) Pabst, G., Grage, S., Danner-Pongratz, S., Jing, W., Ulrich, A. S., Watts, A., Lohner, K., and Hickel, A. (2008) Membrane Thickening by the Antimicrobial Peptide PGLa. Biophys. J. 95 (12), 5779-5788.

(37) Makovitzki, A., Baram, J., and Shai, Y. (2008) Antimicrobial lipopolypeptides composed of palmitoyl Di- and tricationic peptides: in vitro and in vivo activities, self-assembly to nanostructures, and a plausible mode of action. Biochemistry 47, 10630-10636.

(38) Pourmousa, M., and Karttunen, M. (2013) Early stages of interactions of cell-penetrating peptide penetratin with a DPPC bilayer. Chem. Phys. Lipids 169, 85-94.

(39) Rathinakumar, R, and Wimley, W. C. (2008) Biomolecular Engineering by Combinatorial Design and High-Throughput Screening: Small, Soluble Peptides That Permeabilize Membranes. J. Am. Chem. Soc. 130, 9849-9858.

(40) Rathinakumar, R., Walkenhorst, W. F., and Wimley, W. C. (2009) Broad-spectrum antimicrobial peptides by rational combinatorial design and high-throughput screening: the importance of interfacial activity. J. Am. Chem. Soc. 131, 7609-7617.

(41) Wimley, W. C. (2010) Describing the Mechanism of Antimicrobial Peptide Action with the Interfacial Activity Model. ACS Chem. Biol. 5, 905-917. 